\title{
Assistência de Enfermagem a crianças ribeirinhas com parasitoses na Amazônia: Revisão integrativa de literatura
}

Nursing assistance to riverside-living children with parasitisis in the Amazon: An integrative literature review

Asistencia de Enfermería a niños ribereños con parasitosis en la Amazonia: Revisión integrativa de la literatura

Recebido: 13/04/2021 | Revisado: 20/04/2021 | Aceito: 23/04/2021 | Publicado: 09/05/2021

Beatriz Rodrigues Silva ORCID: https://orcid.org/0000-0002-7397-651X Universidade Federal do Pará, Brasil E-mail: br977587@gmail.com

Maria Eduarda Libório Martins ORCID: https://orcid.org/0000-0002-2217-4103 Universidade Federal do Pará E-mail: mliboriomartins@gmail.com

Thamyles da Silva Dias ORCID: https://orcid.org/0000-0002-8797-9025 Universidade Federal do Pará, Brasil

E-mail: thamyles.dias@gmail.com

Jenifer Iris da Costa Martins

ORCID: https://orcid.org/0000-0002-8606-4819

Universidade Federal do Pará, Brasil

E-mail: irisjefmartins@gmail.com

Emely Borges das Neves

ORCID: https://orcid.org/0000-0002-2227-9490

Universidade Federal do Pará, Brasil

E-mail: emelyneves7@gmail.com

Evelyn Sthefane Santos Melo

ORCID: https://orcid.org/0000-0003-0468-8335

Universidade Federal do Pará, Brasil

E-mail: evelyn_melo@outlook.com

Jordy Rodrigues Reis

ORCID: https://orcid.org/0000-0002-1689-7065

Universidade Federal do Pará

E-mail: jordyreus80@gmail.com

Valéria Gabriele Caldas Nascimento

ORCID: https://orcid.org/0000-0002-8900-8388

Universidade Federal do Pará, Brasil

E-mail: valerianascimento5@gmail.com

Wanderson Santiago de Azevedo Júnior

ORCID: https://orcid.org/0000-0001-6125-3261

Universidade Federal do Pará, Brasil

E-mail: wanderson_santiago@hotmail.com

Andressa Tavares Parente

ORCID: https://orcid.org/0000-0001-9364-4574 Universidade Federal do Pará, Brasil E-mail: andressatp@ufpa.br

Pedro Vitor Rocha Vila Nova

ORCID: https://orcid.org/0000-0001-6398-1326 Universidade Federal do Pará, Brasil

E-mail:pedrovn3@gmail.com

Sthefany Borges das Neves

ORCID: https://orcid.org/0000-0001-5185-8216 Centro Universitário do Pará, Brasil

E-mail: sthefany.borges11@gmail.com

Silline Nalane Baia da Silva

ORCID: https://orcid.org/0000-0002-7540-3643

Centro Universitário do Pará, Brasil

E-mail: sillinenalane@outlook.com

Carmem Irany dos Santos Oliveira

ORCID: https://orcid.org/0000-0001-8997-9806

Universidade Federal do Pará, Brasil 


\title{
Resumo
}

Evanildo Lopes Monteiro

ORCID: https://orcid.org/0000-0003-3501-6267 Universidade Federal do Pará, Brasil E-mail: emonteiro@ufpa.br

Este estudo tem como objetivo verificar e analisar os artigos produzidos na literatura científica que dissertam sobre a temática em periódicos nacionais e analisar a assistência de enfermagem em relação ao cuidado com a população infantil com parasitoses. Trata-se de revisão integrativa da literatura na qual foram utilizadas as bases de dados LILACS (Literatura Latino Americana e do Caribe em Ciências da Saúde), SciELO (Scientific Eletronic Library Online) e BDEnf no idioma português no período compreendido de 2010 a 2020. A partir da pesquisa dos dados foram utilizados 10 artigos para que fosse composta a discussão, sendo 3 artigos da LILACS, 5 da SciELO e 2 da BDEnf. A população infantil ribeirinha está mais desprotegida ao contágio por parasitos, devido à precariedade de saneamento básico na região somado a fatores culturais de alimentação e higiene pessoal, desse modo, a assistência de enfermagem tem um papel essencial promovendo a prevenção e tratamento dessas enfermidades, a fim de garantir saúde e bem-estar às crianças.

Palavras-chave: Doenças parasitárias; Infecções; Ecossistema amazônico; Atenção primária à saúde.

\begin{abstract}
This study aims to verify and analyze the articles produced in the scientific literature that discuss the theme in national journals and to analyze nursing care related to the care in child population with parasites. This is an integrative literature review using the LILACS (Latin American and Caribbean Literature in Health Sciences), SciELO (Scientific Electronic Library Online) and BDEnf databases, published in Portuguese from 2010 to 2020. From the data search, 10 articles were used to compose the discussion, 3 articles from LILACS, 5 from SciELO and 2 from BDEnf. The riverside child population is more unprotected from infection by parasites due to the precariousness of basic sanitation in the region, in addition to cultural factors of food and personal hygiene, thus, nursing care has an essential role in promoting the prevention and treatment of these diseases, in order to ensure health and well-being for children.
\end{abstract}

Keywords: Parasitic diseases; Infections; Ecosistema amazónico; Primary health care.

\section{Resumen}

Este estudio tiene como objetivo verificar y analizar los artículos producidos en la literatura científica que disertan sobre la temática en periódicos nacionales y analizar la asistencia de enfermería en relación al cuidado de la población infantil con parásitos. Se trata de revisión integrativa de la literatura en la cual fueron utilizadas (las) bases de datos LILACS (Literatura Latinoamericana y del Caribe en Ciencias de la salud), SCIELO (Scientific Eletronic Library Online) y BDEnf. E en idioma portugués en el período comprendido de 2010 a 2020. A partir de la investigación (de los datos) se usó (fueron utilizados) 10 artículos para componer la discusión, 3 artículos de LILACS, 5 de SciELO y 2 de BDEnf. La población infantil (ribereña) se encuentra más (vulnerable) desprotegida de (al) contagio por parásitos, debido (a) la precariedad del saneamiento básico en la región sumado a factores culturales de alimentación e higiene personal, de ese modo, la asistencia de enfermería tiene un papel esencial promoviendo la prevención y tratamiento de esas enfermedades, a fin de garantizar la salud y el bienestar de los niños.

Palabras clave: Enfermedades parasitarias; Infecciones; Amazonian ecosystem; Atención primaria de salud.

\section{Introdução}

A população ribeirinha habita as bordas dos rios e vive de acordo com as circunstâncias propiciadas pela natureza, como a caçada, a pescaria e o roçado. Diante disso, este meio apresenta entraves relacionados à saúde, principalmente, devido à ausência de serviços de esgoto, coleta de lixo, limpeza pública e água. Além disso, educação sanitária ineficaz e condições desapropriadas de residência são outros fatores que somados aos anteriores favorecem a ocorrência de parasitoses. Por conseguinte, são infecções causadas por parasitas no decorrer da relação do parasitismo, as quais destacam-se as verminoses enterobíase, ascaridíase, teníase, tricuríase e ancilostomíase (Moraes, Souza, Pimentel, Lima \& Maia, 2020; Guimarães et al., 2020).

Calcula-se que as doenças parasitárias prejudiquem mais de três bilhões de indivíduos, acarretando mal-estar e adoecimento em mais de 400 milhões em torno do mundo, sendo que a maioria dos afetados são crianças. Esta classe de infecções estimulam o desenvolvimento de outras enfermidades e agravos à saúde dessas populações, como anemia, irritabilidade, desnutrição, alterações no crescimento, deficiência intelectual e complicações agudas (Belo et al., 2012; Menezes et al., 2020). 
Estima-se que infecções intestinais causadas por helmintos e protozoários afetem cerca de 3,5 bilhões de pessoas, causando enfermidades em aproximadamente 450 milhões ao redor do mundo, a maior parte destas em crianças. Desnutrição, anemia, diminuição no crescimento, retardo cognitivo, irritabilidade, aumento de suscetibilidade a outras infecções e complicações agudas são algumas das morbidades decorrentes. A prevalência de infecções por parasitos intestinais é um dos melhores indicadores do status socioeconômico de uma população e pode estar associada a diversos determinantes, como instalações sanitárias inadequadas, poluição fecal da água e de alimentos consumidos, fatores socioculturais, contato com animais, ausência de saneamento básico, além da idade do hospedeiro e do tipo de parasito infectante (Belo et al., 2012; Sena et al., 2020).

As parasitoses intestinais continuam sendo reconhecidamente elevadas entre as populações de áreas desprovidas de saneamento básico ao redor do mundo. Na Região Amazônica, essa situação constitui um dos principais problemas de saúde pública, especialmente em comunidades ribeirinhas onde as condições de saneamento básico são precárias, inexistindo infraestrutura que viabilize o destino adequado dos dejetos, bem como o acesso à água potável. Desse modo, o uso da água do rio nas atividades domésticas e higiene pessoal representam um risco à saúde da população devido à presença de diferentes patógenos que são veiculados pela água contaminada (Silva et al., 2014; Picanço et al., 2019).

Diante disso, o enfermeiro, como profissional de saúde, assume um papel cada vez mais decisivo e proativo no que se refere à identificação das necessidades de cuidado da população, bem como de medidas para a proteção dos indivíduos contra parasitoses. Além disso, o enfermeiro atua compartilhando o seu conhecimento científico, por meio da ação da lavagem das mãos, sendo esta uma eficiente prática de higiene, a qual deve ser reforçada para a população a fim de distanciar as parasitoses (Belo et al., 2012; Silva et al., 2019; Silva et al., 2020).

A realização dos exames laboratoriais básicos, como rotina de urina, parasitológico das fezes e hemograma, justificase pela contemplação da melhoria da saúde dos moradores das comunidades ribeirinhas, com a função de prevenir e diagnosticar doenças infectoparasitárias (Baptista, Ramos, \& Santos, 2013; Gomes et al., 2016).

Objetiva-se com esse estudo identificar o que se tem produzido na literatura científica que abordam a temática em periódicos nacionais realizando uma revisão integrativa, visando compreender e analisar a assistência de enfermagem em crianças ribeirinhas com parasitoses na Região Amazônica e a forma pela qual a enfermagem contribui para o aprimoramento de sua ferramenta de trabalho.

\section{Metodologia}

Trata-se de revisão integrativa estruturada nas seguintes etapas: 1-formulação do problema; 2-estabelecimento de critérios de inclusão e busca na literatura; 3-definição das informações a serem extraídas da avaliação dos estudos a serem incluídos na revisão; 4-interpretação e apresentação dos resultados. Trata-se de um desenho de pesquisa que busca a apresentação sobre o panorama do conhecimento produzido e o desenvolvimento dessa área de pesquisa, mapeando a produção científica (Mendes, Siqueira \& Galvão, 2008; Sousa, Marques-Vieira, Severino \& Antunes, 2017).

As bases de dados utilizadas para a busca dos estudos incluídos na revisão foram: LILACS (Literatura Latino Americana e do Caribe em Ciências da Saúde); SciELO (Scientific Eletronic Library Online) e BDEnf. E como critérios de inclusão para o estudo, foram selecionados: artigos publicados no idioma português, dos anos de 2010 a 2020, na forma de texto completo que abordassem as temáticas relacionadas ao assunto em questão.

Como estratégia de busca, empregaram-se descritores previamente submetidos aos índices de descritores da DeCS (Descritores em Ciências da Saúde): Doenças Parasitárias; Infecções; Ecossistema Amazônico; Atenção Primária à Saúde.

Após a identificação, realizou-se a seleção dos estudos primários, de acordo com a questão norteadora e os critérios de inclusão previamente definidos. Todos os estudos identificados por meio da estratégia de busca foram inicialmente avaliados por 
meio da análise dos títulos seguidos pela leitura dos resumos e leitura do artigo completo. Nos casos em que os títulos e os resumos não se mostraram suficientes para definir a seleção inicial, procedeu-se à leitura na íntegra da publicação.

Em 1998, estudiosos de enfermagem elaboraram uma classificação hierárquica das evidências para a avaliação de pesquisas ou outras fontes de informação, baseadas na categorização da Agency for Healthcare Research and Quality (AHRQ) dos Estados Unidos da América. A qualidade das evidências é classificada em seis níveis, a saber. Nível 1 - Metanálise de múltiplos estudos controlados. Nível 2 - Estudo individual com delineamento experimental; Nível 3-Estudo com delineamento quase experimental como estudo sem randomização com grupo único pré e pós-teste, séries temporais ou caso-controle; Nível 4 - Estudo com delineamento não experimental como pesquisa descritiva correlacional e qualitativa ou estudos de caso; Nível 5 Relatório de casos ou dado obtido de forma sistemática, de qualidade verificável ou dados de avaliação de programas. Nível 6 opinião de autoridades respeitáveis baseada na competência clínica ou opinião de comitês de especialistas, incluindo interpretações de informações não baseadas em pesquisas (Mendes, Siqueira \& Galvão, 2008).

\section{Resultados}

Inicialmente, realizou-se busca nas bases de dados acima mencionadas, resultando em 53 artigos. Em uma segunda fase de seleção foram lidos os títulos e resumos e excluídos artigos que não atendiam aos critérios de inclusão, resultando na remoção de 31 artigos, sobrando 22 artigos que foram lidos criteriosamente. A partir dessas leituras foram selecionados 13 artigos para compor o estudo, conforme ilustra a figura a seguir: 
Figura 1. Fluxograma de protocolo realizado na pesquisa.

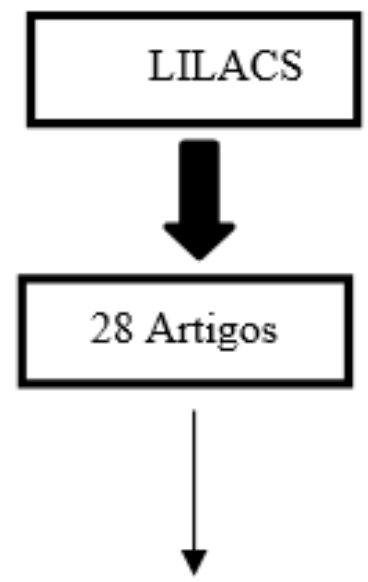

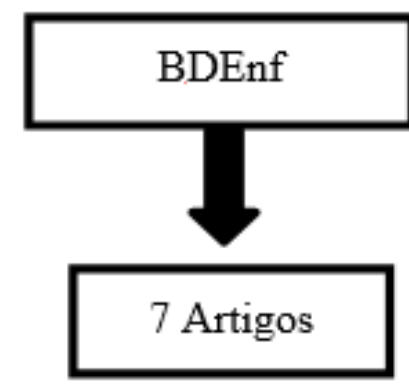

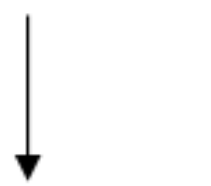

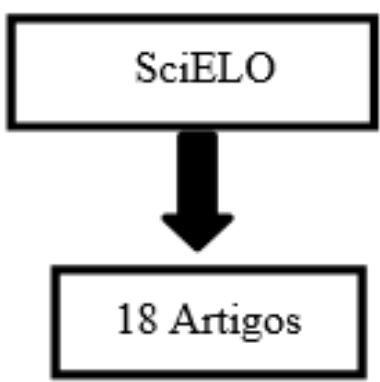

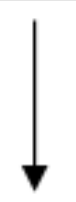

Exclusão de artigos que não atendiam aos critérios de inclusão
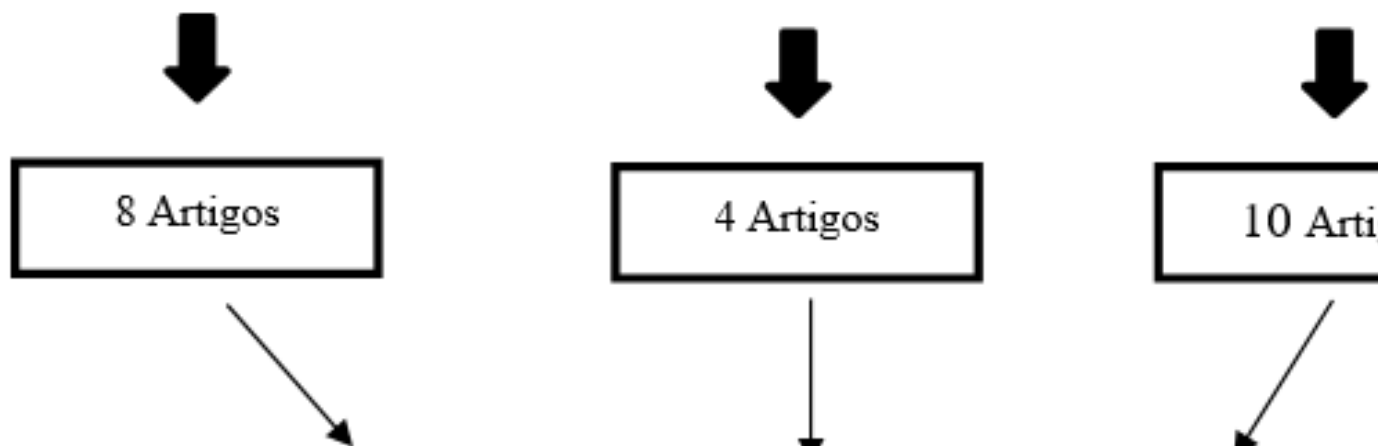

4 Artigos

10 Artigos

Artigos Lidos integralmente

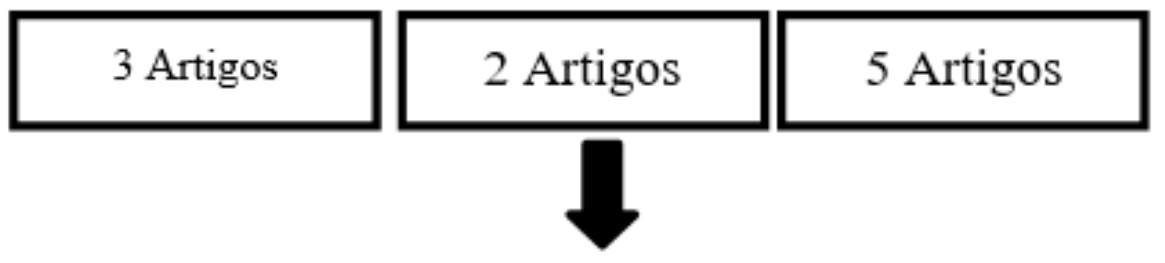

\section{Artigos incluídos no estudo}

Os artigos selecionados estão demonstrados no quadro abaixo, que contém informações de título, autoria, ano e resultados. Tendo-se recorrido à análise das evidências apresentadas para a discussão. 
Quadro 1. Síntese dos principais achados dos artigos selecionados.

\begin{tabular}{|c|c|c|}
\hline Publicação & Autor/Ano & Resultado \\
\hline $\begin{array}{l}\text { Fatores associados à } \\
\text { ocorrência de parasitoses } \\
\text { intestinais em uma população } \\
\text { de crianças e adolescentes }\end{array}$ & $\begin{array}{l}\text { Belo et all, } \\
2012\end{array}$ & $\begin{array}{l}\text { Estudo que analisar a prevalência de infecções por parasitos intestinais em crianças } \\
\text { e adolescentes matriculados em escolas de ensino fundamental no município de } \\
\text { São João del-Rei, A prevalência geral de parasitoses intestinais encontrada no } \\
\text { estudo foi de } 29 \% \text {, isto é, } 335 \text { alunos infectados com, pelo menos, um parasito }\end{array}$ \\
\hline
\end{tabular}

Ocorrência de
enteroparasitoses em
$\begin{aligned} & \text { comunidades ribeirinhas do } \\ & \text { Município de Igarapé Miri, } \\ & \text { Estado do Pará, Brasil }\end{aligned}$

Silva et all, 2015

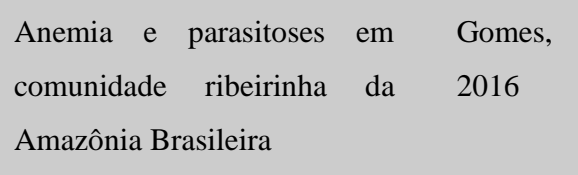

Parasitoses intestinais e baixos

Ferraz, índices de Gini em Macapá 2015

(AP) e Timon (MA), Brasil
Os resultados revelaram positividade em $94,5 \%$, ressaltando que em $57,17 \%$ dos casos positivos estavam biparasitados ou poliparasitados. Os helmintos foram os mais frequentes, destacando-se: Ascaris lumbricoides (57,14\%) e Trichuris trichiura $(41,76 \%)$. Entre os protozoários, os mais frequentes foram Entamoeba spp. $(36,26 \%)$, Giardia intestinalis $(24,17 \%)$ e Iodamoeba butschlii $(23,08 \%)$. Os resultados obtidos revelam a necessidade de implementação de políticas públicas voltadas para programas do governo que visem melhorias no saneamento e saúde nas comunidades ribeirinhas

Estudo que analisa os resultados dos exames laboratoriais básicos, associando os achados com as principais patologias associadas à pobreza e à vulnerabilidade social. Os resultados obtidos demonstraram uma alta prevalência de parasitoses e uma prevalência moderada de anemia, relacionando-se à vulnerabilidade social das comunidades ribeirinhas

As parasitoses intestinais ainda constituem um sério problema de Saúde Pública, devido a sua alta prevalência na população, associação com diversos fatores ambientais e seus complexos ciclos epidemiológicos. Este estudo faz um levantamento sobre a ocorrência de enteroparasitoses em duas cidades — Timon (MA) e Macapá (AP). Verificou-se associação entre a prevalência de parasitoses e os baixos índices de Gini. O controle dessas doenças necessita de políticas públicas envolvendo educação sanitária e ambiental, além de melhor distribuição de renda.

$\begin{array}{lll}\begin{array}{l}\text { Prevalência de parasitoses } \\ \text { intestinais em crianças do }\end{array} & \begin{array}{l}\text { Escobar- } \\ \text { Pardo, }\end{array} & \begin{array}{l}\text { Este estudo tem por objetivo avaliar a prevalência da parasitose intestinal em } \\ \text { crianças indígenas de } 2 \text { a } 9 \text { anos. Para a realização do exame protoparasitológico, } \\ \text { Parque Indígena do Xingu }\end{array} \\ \text { foram convidadas todas as crianças de } 2 \text { a } 9 \text { anos, de seis aldeias localizadas no } \\ \text { Médio e Baixo Xingu. Não houve diferença significativa entre as idades médias } \\ \text { das crianças provenientes das seis aldeias. Resultaram positivas para a presença de } \\ \text { parasitas, 97,5\% (198/202) e 96,1\% (98/102) na primeira e segunda coletas, } \\ \text { respectivamente, sem associação estatística entre a idade. }\end{array}$




$\begin{array}{ll}\text { Perfil parasitário de uma } & \text { Inagaki, } \\ \text { comunidade adscrita à unidade } & 2014 \\ \text { de saúde da família } & \end{array}$
de saúde da família

Este estudo descreve o perfil parasitário de uma comunidade assistida pela Estratégia de Saúde da Família. Um quarto dos pacientes possuíam pelo menos uma enteroparasitose. Infestação por Entamoeba histolytica, Giardia lamblia e Ascaris lumbricoides foram as mais frequentes. Dos indivíduos parasitados a maioria era do sexo feminino, na faixa etária de 20-60 anos; entre os homens, a infestação foi mais frequente nas crianças e nos adolescentes; dentre as gestantes, a maior ocorrência foi de Entamoeba histolytica.

$\begin{array}{lllll}\text { Promoção de educação em } & \text { Tavares, } & \text { Artigo que descreve a promoção de educação em saúde para prevenção de } \\ \text { saúde para a prevenção de } & 2010 & \text { parasitoses, bem como persuadir a vivência das medidas de prevenção pelos } \\ \text { parasitoses: } \quad \text { relato de } & & \text { sujeitos. Observou-se o conhecimento prévio dos sujeitos sobre algumas } \\ \text { experiência } & & \text { parasitoses, uma boa interação acerca da temática, dando ênfase ao compromisso } \\ \text { de vivenciar as medidas de prevenção }\end{array}$

Cuidado infantil e infecções Andrade, parasitárias

\section{3}

Dentre as infecções parasitárias observadas neste grupo populacional, verificou-se a prevalência de $44,50 \%$ para as enteroparasitoses e 31,20\% para ectoparasitoses, especificamente pediculose e escabiose. Houve uma prevalência maior de monoparasitismo $(29,3 \%)$, em relação às infecções concomitantes $(12,7 \%$ de biparasitismo e $2,5 \%$ de poliparasitismo). Dentre os enteroparasitos diagnosticados, os helmintos mais frequentes foram Ascaris lumbricoides e entre os protozoários Giardia lamblia

\begin{abstract}
Promoção de saúde na perspectiva da prevenção de doenças parasitárias por meio da educação em saúde com escolares do ensino fundamental
\end{abstract}

\section{Enteroparasitoses na}

População Infantil sua

Prevalência e os Modelos de

Decisão Utilizados: Revisão

Sistemática
O artigo relata a experiência de uma ação de educação em saúde com escolares do ensino fundamental de uma escola pública em zona rural. Foi realizada uma ação de educação em saúde com o envolvimento de 30 alunos, 03 professores e 09 pais. Foi identificado que as crianças estão expostas a risco de contaminação por parasitas, visto as condições sanitárias que habitam, evidenciando a necessidade da ampliação de ações de educação em saúde acerca da importância da prevenção das parasitoses.
Dias, 2018

Este artigo tem por objetivo identificar a prevalência de enteroparasitoses na população infantil e principais modelos de decisão utilizados na tomada de decisão. Ascaris lumbricoides; Ancylostomidae; Trichuris trichiura; Giardia lamblia; Entamoeba coli; Endolimax nana, são os tipos que mais afetam a população. As análises estatísticas estão sendo feitas de forma pouco detalhada, quanto ao seu delineamento.

Fonte: Autores da pesquisa (2021).

\section{Discussão}

Os achados podem ser entendidos a partir do que refere o autor, o qual aponta as parasitoses intestinais como um complexo problema de saúde pública. Destacando que parte das doenças que acometem crianças em países subdesenvolvidos tem como causa básica as infecções parasitárias. Os agravos relacionam-se, sobretudo, às precárias condições socioeconômicas associadas ao baixo nível de escolaridade e ao pouco conhecimento das condições higiênicas e sanitárias da população. Desse modo, em relação à infecção por parasitos, destaca-se a transmissão fecal como uma questão de grande relevância, pois em 
comunidades mais remotas os dejetos são feitos no chão, o que proporciona o contágio da superfície e da água, além de evidenciar a ausência da vigilância sanitária. Este processo ocorre pela disposição de ovos e larvas expedidos nas fezes, os quais progridem até a sua fase infectante (Silva et al., 2014).

Os protozoários intestinais transmitem-se principalmente pela contaminação fecal através das mãos, da água ou dos alimentos. Com relação às variáveis sociodemográficas, verifica-se que, por exemplo, no município de Igarapé-Miri os helmintos foram os mais frequentes, destacando-se: Ascaris lumbricoides (57,14\%) e Trichuris trichiura (41,76\%). Esse resultado obtido demonstra que há uma alta prevalência de parasitoses na população em questão, sendo necessária a implementação de políticas públicas voltadas para melhorias no saneamento e saúde deste município (Silva et al., 2014; Escobar-Pardo et al., 2010).

No Brasil, a ocorrência de parasitoses varia bastante nas diferentes regiões, relacionada ao desenvolvimento econômico. Na região amazônica, principalmente, nas comunidades ribeirinhas as condições de saneamento básico são precárias e é inexistente infraestrutura que dê destino adequado aos dejetos jogados ao rio. Sendo assim, acaba por acarretar riscos à saúde dessa população, já que, há presença de diversos patógenos na água contaminada, sendo a mesma utilizada para atividades domésticas e higiene pessoal. Nessa perspectiva, verifica-se que o acesso dessa população ao atendimento básico é bastante difícil. O deslocamento torna-se custoso, principalmente, quando o atendimento é demorado ou até mesmo quando não se consegue atendimento no mesmo dia (Belo et al., 2012; Silva et al., 2014).

Um estudo feito por um grupo de alunos da UFPA, revela que em crianças de comunidades ribeirinhas foram encontrados até mais de uma espécie parasitária. Parasitas esses que, podem acabar causando anemia, diarréia, má absorção de nutrientes, levando a um possível dano no processo cognitivo. As enteroparasitoses são mais recorrentes nas crianças em idade escolar o que pode justificar a alta prevalência nas idades de 1 a 12 anos de idade. Essas infecções geram uma deficiência de aproveitamento escolar em crianças e jovens (Gomes et al., 2016).

Os enteroparasitos possuem grande prevalência entre as crianças, em maioria, as doenças causadas são negligenciadas como problema de saúde pública, ainda que possam causar relevantes danos à criança. Em uma análise, Cavalcante identificou - se os enteroparasitos mais prevalentes diante a estudos sendo estes: Ascaris lumbricoides; Ancylostomidae; Trichuris trichiura; Giardia lamblia; Entamoeba coli; Endolimax nana (Cavalcante, Melo, Dantas, Coelho \& Lima, 2015).

De acordo com o autor, o cuidado e as infecções parasitárias em crianças e os reflexos da estruturação familiar, tendo em vista dados a cerca sobre água, situação de moradia e socioeconômica, conferindo prevalência de 44,5\% para enteroparasitos (ascaridíase/21,7\%) e 31,2\% para ectoparasitos (pediculose/18,2\%), de condições de moradia inadequadas além de acentuar para as questões de higiene e educação infantil e nos reflexos que traz consigo (Andrade et al., 2013).

Entre os fatores de risco associados às altas prevalências de parasitoses, além da ausência de saneamento básico, estão a cultura, que na maioria das vezes é comum, comer com as mãos, defecar no ambiente- normalmente a deposição de fezes é na área de peridomicílio, não usar calçados, dentre outros hábitos higiênicos. Em contraponto, outro estudo apresenta uma redução de casos de infecções por parasitoses, com a instalação sanitária no domicílio e uso de filtro de água, com a necessidade de que os próprios moradores realizem tratamento adicional na água que irá consumir (Belo et al., 2012; Escobar-Pardo et al., 2010).

Pesquisas também mostraram que falta educação em saúde como medida de prevenção primária às parasitoses, associadas aos fatores sociais e educativos, assim como, medidas de Saúde Pública e ações de educação para a saúde nas áreas endêmicas, onde o tratamento público é inexistente ou esteja apresentando falhas. A enfermagem enquanto protagonista no cuidado a ser prestado às populações ribeirinhas possui, enquanto parte da assistência, a promoção e prevenção à saúde, envolvendo educação sanitária e ambiental como aborda Tavares a promoção de educação em saúde para prevenção de parasitoses (Ferraz et al., 2014).

Meninos e meninas em crescimento necessitam de condutas instrutivas acerca de práticas de higiene adequadas e adoção de medidas que potencializam a qualidade de vida dos mesmos. Desse modo, o contágio por infecções parasitárias será 
minimizado, pois indivíduos esclarecidos tendem a exercer ações menos prejudiciais à saúde individual e coletiva (Dias, Oliveira, Teixeira, Anjos \& Lima, 2018).

Conforme o autor, as crianças mais atingidas por parasitos estão na faixa etária de dois a quatro anos, justamente no ciclo marcado pela oralidade, além de que nesse período as crianças geralmente já estão andando e se relacionando melhor umas com as outras, o que explica as ações de compartilhar brinquedos que vão à boca naturalmente e ter curiosidade para experimentar novos alimentos. A partir disso, a probabilidade dessa população entrar em contato com parasitas por meio da cavidade oral é grande (Andrade et al., 2013).

As crianças acometidas por doenças parasitárias possuem manifestações comuns a outras enfermidades, como êmese, problema de concentração, mudança no padrão de sono e sentimento de frustração ou raiva. Os mais diretamente relacionados aos parasitos são: diarreia por mais de três meses, desnutrição e a diminuição dos leucócitos, agentes imprescindíveis para que o sistema imunológico realize sua função adequadamente (Andrade et al., 2013).

A partir de estudos que vêm sendo conduzidos nessa região, torna-se evidente a complexa relação existente entre o ecossistema e a população da Amazônia, a qual sugere um desafio: tornar plenas as condições sanitárias da maioria dos indivíduos residentes nesta área que enfrentam diversas dificuldades em relação ao acesso aos serviços de saúde, as quais podem ser explicadas tanto pela falta de estrutura física quanto a de material humano, bem como o elevado custo de estadia nos grandes centros urbanos, os quais são preferencialmente procurados por concentrarem os melhores serviços de saúde. Ademais, segundo o autor, oportunidades como palestras, discussões e rodas de conversa, nas quais se discutem hábitos coerentemente saudáveis em educação em saúde nas áreas mais remotas e de difícil acesso é de natureza enriquecedora, possibilitando que as problemáticas trazidas à discussão sejam capazes de desafiá-los a construir uma "ponte" entre conhecimentos teóricos, práticos e a sua aplicação para solucionar os problemas de saúde. Portanto, a extensão de atividades de instituições que desempenham um papel social e responsável considerável, naturalmente, faz toda a diferença no sistema, sobretudo em regiões mais precárias (Andrade et al., 2013).

\section{Considerações Finais}

É de suma importância que os governantes e a sociedade lancem um olhar mais humano para as diferentes realidades que compõem o estado do Pará, pois a população ribeirinha precisa de mais atenção. Como foi observado no estudo, os resultados revelaram que a maior prevalência de parasitoses intestinais foi identificada em crianças que vivem em condições precárias, às quais estão associadas a diferentes fatores, entre eles: hábitos de higiene pessoal, cuidados no preparo e a forma de consumo de alimentos, uso e manipulação da água nos domicílios, além da ausência de saneamento básico na região.

A partir disso, é imprescindível que seja realizada uma orientação para esses indivíduos compreenderem que a principal forma de transmissão destes agentes ocorre pela via fecal-oral, por meio do consumo de alimentos e água contaminados com ovos e cistos de parasitas. Esse entendimento sobre o processo de saúde-doença contribui com a promoção da saúde e bem-estar dessa parcela importante da população e lhes oferecem ferramentas para transformar a realidade em que estão inseridos. Além disso, cabe ao Estado garantir saúde e bem estar a estes cidadãos, por meio da aplicação das políticas públicas já existentes.

Em síntese, a assistência de enfermagem contribui de modo significativo na vida deste povo, auxiliando e orientando em rotinas diárias, atentando principalmente para a particularidade de cada sujeito, para que ocorra a prevenção e tratamento dessa patologia da melhor forma, uma vez que esta atribuição da enfermagem é fundamental para a melhoria da qualidade de vida dessa população.

Ademais, sugerimos que os estudos acerca das populações ribeirinhas na Amazônia sejam intensificados, a fim de aumentar a visibilidade sobre esses povos e, consequentemente, a atenção e o cuidado. Desse modo, mais trabalhos poderão ser desenvolvidos e publicados e o conhecimento expandido. 


\section{Referências}

Andrade, A. S. A., Carvalho, C. D., Brito, A. M. G., Jeraldo, V. L. S., Oliveira, C. C. C., \& Melo, C. M. (2013). Cuidado infantil e infecções parasitárias/Child care and parasitic infections. Ciência, Cuidado e Saúde, 12(2), 257-265.

Baptista, A. B., Ramos, L. da S., \& Santos, H. A. G. (2013). Prevalência de enteroparasitos e aspectos epidemiológicos de crianças e jovens do município de Altamira - PA. Revista de Pesquisa em Saúde, 14(2), 77-80.

Belo, V. S., Oliveira, R. B. de., Fernandes, P. C., Nascimento, B. W. L., Fernandes, F. V., Castro, C. L. F., Santos, W. B. dos., \& Silva, E. S. (2012). Fatores associados à ocorrência de parasitoses intestinais em uma população de crianças e adolescentes. Revista Paulista de Pediatria, 30(2), $195-201$.

Cavalcante, U. M. B., Melo, S. A. L. de., \& Lima, C. M. B. L. (2015). Enteroparasitoses na população infantil sua prevalência e os modelos de decisão utilizados: revisão sistemática. Saúde e Pesquisa, 8(3), 585-590.

Cavalcante, U. M. B., Melo, S. A. L. de., Dantas, S. H., Coelho, H. F. C., \& Lima, C. M. B. L. (2016). Avaliação da Qualidade de Vida de pacientes com enteroparasitoses atendidos num Hospital Escola de João Pessoa-PB utilizando um Modelo de Regressão Logística. Tempus Actas de Saúde Coletiva, 10(2), 275 .

Dias, E. G., Oliveira, C. K. N. de., Teixeira, J. A. L., Anjos, A. K. T. dos., \& Lima, J. A. D. (2018). Health promotion in the perspective of the prevention of parasitic diseases through education in health with schools of fundamental Education. Revista de Epidemiologia e Controle de Infecção, 8(3), $283-285$.

Escobar-Pardo, M. L., Godoy, A. P. O. D., Machado, R. S., Rodrigues, D., Neto, U. F., \& Kawakami, E. (2010). Prevalência de parasitoses intestinais em crianças do Parque Indígena do Xingu. Jornal de pediatria, 86(6), 493-496.

Ferraz, R. R. N., Barnabé, A. S., Porcy, C., D'Eça, A., Jr., Feitosa, T., \& Figueiredo, P. D. M. (2014). Parasitoses intestinais e baixos índices de Gini em Macapá (AP) e Timon (MA), Brasil. Cadernos Saúde Coletiva, 22(2), 173-176.

Gomes, K. M., Cerqueira, L. E., Sarges, E. S., Souza, F. G., Ribeiro, C. H. M. A., \& Melo, M. F. C. (2016). Anemia e parasitoses em comunidade ribeirinha da Amazônia brasileira. Rev Bras Anal Clin, 48(4), 389-93.

Guimarães, A. F., Barbosa, V. L. M., Silva, M. P. da., Portugal, J. K. A., Reis, M. H. da S., \& Gama, A. S. M. (2020). Acesso a serviços de saúde por ribeirinhos de um município no interior do estado do Amazonas, Brasil. Revista Pan-Amazônica de Saúde, 11, e202000178.

Mendes, K. D. S., Silveira, R. C. D. C. P., \& Galvão, C. M. (2008). Revisão integrativa: método de pesquisa para a incorporação de evidências na saúde e na enfermagem. Texto \& contexto-enfermagem, 17(4), 758-764.

Menezes, R. C. de, Jr., Lima, C. A. A. de, Jr., Marinho, I. G., Braga, K. H. de M., Nascimento, R. O. do, dos Santos Calandrini, T. D. S., Melo, D. P. S., Sacramento, B. P., Andrade, R. F. de., \& Menezes, R. A. de O. (2020). Enteroparasitoses, anemia e estado nutricional de uma população ribeirinha no estado do Amapá. Revista Eletrônica Acervo Saúde, 12(5), e2841-e2841.

Moraes, G. de C., Souza, G. H. da S., Pimentel, K. B., Lima, K. N., \& Maia, G. de O. G. (2020). Tópicos multidisciplinares em ciências biológicas 4 [recurso eletrônico] - Organizador Edson da Silva (pp. 27-30): Atena.

Picanço, N. J. de A., Machado, N. M. M., Nunes Filho, O. P., Monteiro, A. O. S., Kalif, L. da C., Nunes, D. M., Lima, J. J. C. de, Felisberto, D. V. D., Santos, J. F. A, Récio, C. S., Freire. M. L. F., Santos, T. O., Silva. R. A., Furlaneto I. S., \& Dantas, R. T. da S. (2019). Prevalência de enteroparasitoses em usuários na faixa etária de 2 a 12 anos atendidos por uma Unidade de Saúde da Família do município de Belém, Pará. Revista Eletrônica Acervo Saúde, (33), e1321-e1321.

Sena, L. W. P. de., Pantoja, C. de S. C., Souza, D. A. S. de., Palheta, S. S. R., Mello, A. G. N. C., Vieira, J. L. F., Arruda, J. E. G., \& Moreira, M. P. (2020). Prevalência de enteroparasitose em comunidade ribeirinha do estado do Pará, Brasil. Revista Eletrônica Acervo Saúde, 12(11), e4710-e4710.

Silva, A. K., Vasconcelos, B. M., Carvalho, K. G. de., Nery, M. T. R., Ferreira, N. G., Santos, N. S. P. dos, Sá, R. K. A. de, Andrade, M. do S. N. de, Castro, A. P. R. de., \& Medeiros, K. M. F. (2019). Intervenção educativa sobre higienização das mãos para crianças na prevenção de parasitoses. Revista Interfaces, 7(1), $210-214$.

Silva, A. M. B. da., Bouth, R. C., Costa, K. S. da., Carvalho, D. C. de., Hirai, K. E., Prado, R. R., Araújo S. G., Pereira A. C. de L., \& Ribeiro, K. T. S. (2014). Ocorrência de enteroparasitoses em comunidades ribeirinhas do Município de Igarapé Miri, Estado do Pará, Brasil. Revista Pan-Amazônica de Saúde, 5(4), 77 .

Silva, M. P. da., Lima, P. A. V., Pinheiro, W. S., Paz, M. C. D., Dantas, F. M., \& Monteiro, C. E. B. (2020). Assistência de enfermagem no contexto amazônico: vivências de acadêmicos em uma Unidade Básica de Saúde Fluvial. Brazilian Journal of Health Review, 3(5), 12169-12177.

Sousa, L. M. M. de., Marques-Vieira, C. M. A., Severino, S. S. P., \& Antunes, A. V. (2017). A metodologia de revisão integrativa da literatura em enfermagem. Revista Investigação em Enfermagem, 21(2), 17-26.

Tavares, J. S., \& Rodrigues, W. F. G. (2017). Promoção de educação em saúde para a prevenção de parasitoses: relato de experiência. Revista de Enfermagem UFPE On line, 11(8), 3167-3170. 